\title{
Analytic insights on the information content of new observables
}

\author{
Wei-Chia Chen \\ Simons Center for Quantitative Biology, Cold Spring Harbor Laboratory, Cold Spring Harbor, NY 11724, USA

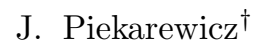 \\ Department of Physics, Florida State University, Tallahassee, FL 32306, USA
}

(Dated: June 16, 2020)

\begin{abstract}
Uncertainty quantification has emerged as a rapidly growing field in nuclear science. Theoretical predictions of physical observables often involve extrapolations to regions that are poorly constrained by laboratory experiments and astrophysical observations. Without properly quantified theoretical errors, such model predictions are of limited value. Also, one often deals with theoretical constructs that involve fundamental quantities that are not accessible to experiment or observation. Particularly relevant in this context is the pressure of pure neutron matter. In this contribution we develop an analytic framework to answer the question of "How can new data reduce uncertainties of current theoretical models?"'P.-G. Reinhard and W. Nazarewicz, Phys. Rev. C81, 051303(R) (2010)]. Simple and insightful expressions are obtained to quantify the impact of one or two new observables on theoretical uncertainties in two critical quantities: the slope of the symmetry energy at saturation density and the pressure of pure neutron matter at twice nuclear matter saturation density.
\end{abstract}

Introduction. In the last few years we have witnessed historical discoveries in astronomy with far-reaching implications in nuclear astrophysics. First, the gravitational wave detection of the binary neutron star merger GW170817 [1] together with its associated electromagnetic counterpart [2] are providing answers to some of the most fundamental questions animating nuclear science today [3, 4. Second, pulsar-timing observations of the millisecond pulsar J0740+6620 by Cromartie and collaborators yielded the heaviest neutron star mass reported to date: $M=2.14_{-0.09}^{+0.10} M_{\odot}[5]$. Finally, the first simultaneous determination of the mass and radius of a neutron star (J0030+0451) was reported by both the Maryland [6] and Amsterdam collaborations [7. Using the Neutron star Interior Composition Explorer (NICER) aboard the international space station, pulse-profile modeling of the thermal emission from the pulsar's hot spots revealed a mass of about $1.4 M_{\odot}$ and a radius of nearly $13 \mathrm{~km}$, with a $\pm 10 \%$ uncertainty in both quantities.

Whereas electromagnetic- and gravitational-wave detections are providing powerful constraints on the equation of state (EOS) at densities above nuclear matter saturation density, measurements of nuclear observablessuch as neutron skins, electric dipole polarizabilities, and heavy-ion collisions - constrain the EOS near saturation density; see Refs. [8] 10] and references contained therein. It is this compelling connection between "heaven and earth" that promises unprecedented advances in the quest to determine the equation of state.

Besides these remarkable advances in nuclear astrophysics, uncertainty quantification has seen significant growth during the last decade. One of the earliest appeals to the theoretical community was issued by the Editors of the Physical Review A who stated: "it is all too often

\footnotetext{
* chenw@cshl.edu

$\dagger$ jpiekarewicz@fsu.edu
}

the case that the numerical results are presented without uncertainty estimates" 11. The nuclear theory community has responded to the challenge with a large number of publications that have addressed not only the critical role of theoretical uncertainties, but also the wealth of information that is contained in the study of statistical correlations among observables [12 22].

Our present work is motivated by the decade-old paper by Reinhard and Nazarewicz [13]. The paper poses two important questions on the uniqueness and usefulness of a new observable: (i) Considering the current theoretical knowledge, what novel information does new measurement bring in? and (ii) How can new data reduce uncertainties of current theoretical models? In an effort to illuminate this numerical procedure, we propose to answer these questions analytically and extend the argument to the case of adding more than one observable. Although by necessity some approximations will be required, our goal is to provide valuable insights while retaining accuracy. In particular, the formalism allows us to readily assess situations that often involve limited experimental resources: should one attempt a single highprecision measurement or would it be better to attempt two (or more) measurements with less precision [23].

A particular interesting and topical example is the slope of the symmetry energy at saturation density $L$, a quantity that is closely connected to the pressure of pure neutron matter at saturation density. The slope of the symmetry energy plays an important role in areas as diverse as nuclear structure, heavy-ion collision, neutron star structure, and supernova explosion [8 10]. Given that $L$ is not a physical observable, a determination of $L$ requires theoretical modeling. Yet, such a determination is hindered by the fact that different theoretical models tend to predict widely different values. However, the neutron skin thickness of ${ }^{208} \mathrm{~Pb}$ - defined as the difference in the root mean square radius between its neutron and proton distributions - has been shown to be strongly 
correlated to $L$ 24 27]. The pioneering Lead Radius EXperiment (PREX) at the Jefferson Laboratory (JLab) has provided the first model-independent evidence in favor of a neutron-rich skin in ${ }^{208} \mathrm{~Pb}[28,29]$. Moreover, the recently completed PREX-II campaign aims to improve on the original PREX result by about a factor of three, thereby reaching a precision of about $0.06 \mathrm{fm}$ on the neutron radius of ${ }^{208} \mathrm{~Pb}\left(R_{n}^{208}\right)$. Scheduled to run immediately after PREX-II, the Calcium Radius EXperiment (CREX) aims to determine the neutron radius of ${ }^{48} \mathrm{Ca}$ $\left(R_{n}^{48}\right)$ with a $0.03 \mathrm{fm}$ precision [30]. Finally, the planned P2 experiment at the Mainz Energy recovery Superconducting Accelerator (MESA) will become the paradigm of a new generation of high-precision parity-violating electron-scattering experiments 36. Within the scope of the P2 setup, the Mainz Radius EXperiment (MREX) will determine $R_{n}^{208}$ and $R_{n}^{48}$ with a precision of $0.03 \mathrm{fm}$ and $0.02 \mathrm{fm}$, respectively [10. How will all these measurements impact the theoretical uncertainty in $L$ is a question that the formalism developed here is ideally positioned to answer.

Formalism. Nuclear energy density functionals are calibrated by minimizing an objective "chi-square" function

$$
\chi_{N}^{2}(\mathbf{p})=\sum_{n=1}^{N} \frac{\left(\mathcal{O}_{n}^{\mathrm{th}}(\mathbf{p})-\mathcal{O}_{n}^{\exp }\right)^{2}}{\sigma_{n}^{2}},
$$

where $N$ is the number of experimental observables included in the fit, $\mathbf{p}$ is a point in the $F$-dimensional parameter space, $\mathcal{O}_{n}^{\exp }$ represents the central value of the measured observables with an associated uncertainty $\sigma_{n}$, and $\mathcal{O}_{n}^{\text {th }}(\mathbf{p})$ is the corresponding theoretical prediction. Although in principle $\sigma_{n}$ is associated with the experimental uncertainty, in practice it is often supplemented with a "theoretical" contribution. The main reason behind this choice is to prevent the model from being biased toward those observables that are measured with great precision, such as binding energies.

The distribution of model parameters $\mathbf{p}$ informed by the $N$ experimental observables is encoded in the likelihood function that represents the relative probability that a set of model parameters $\mathbf{p}$ reproduces the given experimental data. However, in general an arbitrary point in parameter space will often produce unphysical results or will lead to calculations that fail to converge. To overcome this situation one introduces a physically reasonable domain of parameters by incorporating one's own biases and intuition.

Assuming that $\chi_{N}^{2}(\mathbf{p})$ is minimized at the point $\mathbf{p}_{0}$, one can Taylor-expand Eq.(1) around $\mathbf{p}_{0}$ to obtain

$$
\begin{aligned}
\chi_{N}^{2}(\mathbf{p}) & =\chi_{N}^{2}\left(\mathbf{p}_{0}\right)+\delta p_{i}\left(\frac{\partial \chi_{N}^{2}}{\partial p_{i}}\right)_{0}+\frac{1}{2} \delta p_{i} \delta p_{j}\left(\frac{\partial^{2} \chi_{N}^{2}}{\partial p_{i} \partial p_{j}}\right)_{0}+\ldots \\
& \equiv \chi_{N}^{2}\left(\mathbf{p}_{0}\right)+\delta p_{i} M_{i j} \delta p_{j}+\ldots
\end{aligned}
$$

where $\delta p_{i}=\left(\mathbf{p}-\mathbf{p}_{0}\right)_{i}$ and a summation over repeated indices is assumed. The first term in the above expression is a constant while the second term vanishes at the minimum. Hence, the landscape near the minimum is entirely controlled by the curvature matrix $M$ (i.e., the matrix of second derivatives) which by construction is positive definite. In terms of the curvature matrix the covariance between two quantities $A$ and $B$ is given by $[13,33$,

$$
\operatorname{cov}(A, B)=\sum_{i, j=1}^{F}\left(\frac{\partial A}{\partial p_{i}}\right)_{0} M_{i j}^{-1}\left(\frac{\partial B}{\partial p_{j}}\right)_{0},
$$

where $M^{-1}$ is the covariance matrix. Given that the curvature matrix $M$ is positive definite, the existence of its inverse is guaranteed. If $A=B$, then the above expression yields the variance of $A: \tau_{A}^{2}=\operatorname{cov}(A, A)$. Note that we are using $\tau$ rather than $\sigma$ to denote the theoretical uncertainty; $\sigma$ is reserved to denote the experimental error. Finally, the Pearson correlation coefficient is given by

$$
\varrho(A, B)=\frac{\operatorname{cov}(A, B)}{\sqrt{\operatorname{cov}(A, A) \cdot \operatorname{cov}(B, B)}} .
$$

A value of $|\varrho(A, B)|=1$ implies that the two quantities are perfectly correlated. If instead $\varrho(A, B)=0$, then the two quantities are totally uncorrelated. In the particular case of the slope of symmetry energy $L$, we regard its central value and its associated theoretical uncertainty $\tau_{L}$ as our prior knowledge, given the $N$ experimental measurements. As new observables are incorporated into the data set, our knowledge of $L$ improves.

Information content of one new observable. In this section we develop an analytic formalism to assess the information content of a new observable. The objective function resulting from the addition of one new observable is obtained by simply enlarging the sum displayed in Eq.(1). There are (at least) two well-known approaches on how to estimate the impact of a new measurement. The simplest, yet numerically most costly, approach involves an entire new calibration of the model parameters [13]. A numerically less costly approach relies on Bayesian inference [31, 32]. In this case one updates the distribution of model parameters using only the new available information. In the jargon of Bayesian inference, "today's posterior is tomorrow's prior". Given that the prior distribution is exclusively a function of the model parameters, the likelihood function requires theoretical predictions for only the new observable. We introduce here a third approach that is entirely analytic. To do so, we now make the central assumption underlying our work: the minimum of the augmented objective function $\chi_{N+1}^{2}$ remains fixed at $\mathbf{p}_{0}$. Under this assumption, the second derivative of the augmented chi-square function becomes

$$
\left(\frac{\partial^{2} \chi_{N+1}^{2}}{\partial p_{i} \partial p_{j}}\right)_{0}=\left(\frac{\partial^{2} \chi_{N}^{2}}{\partial p_{i} \partial p_{j}}\right)_{0}+\frac{2}{\sigma_{I}^{2}}\left(\frac{\partial \mathcal{O}_{I}^{\text {th }}}{\partial p_{i}}\right)_{0}\left(\frac{\partial \mathcal{O}_{I}^{\text {th }}}{\partial p_{j}}\right)_{0},
$$

where $\mathcal{O}_{I}$ is the new experimental observable added to the previous data set. This relation implies that the augmented curvature matrix may be written as

$$
\mathcal{M}_{i j}=M_{i j}+\Gamma_{i j}
$$


where the symmetric matrix $\Gamma$ has been defined as

$$
\Gamma_{i j}=\gamma_{i} \gamma_{j}=\left[\frac{1}{\sigma_{I}}\left(\frac{\partial \mathcal{O}_{I}^{\text {th }}}{\partial p_{i}}\right)_{0}\right]\left[\frac{1}{\sigma_{I}}\left(\frac{\partial \mathcal{O}_{I}^{\text {th }}}{\partial p_{j}}\right)_{0}\right]
$$

Given that the entire statistical framework relies on the inverse of the curvature matrix, we must address how to invert the sum of two matrices, as given in Eq.(6). In general, the inverse of a sum of two matrices is not guaranteed to exist even when both matrices are invertible. However, Miller has shown that in the case that one of the matrices has an inverse and the other one is a matrix of rank 1, then the inverse exists 34. In our case the augmented matrix $\mathcal{M}$ has been written as the sum of an invertible, positive definite matrix $M$ plus a rank-1 matrix $\Gamma$ constructed from the outer product of the vector $\gamma$ defined above. Note that the rank of a symmetric matrix is the dimension of its column (or row) space. The inverse of $\mathcal{M}$ is then given by [34]:

$$
\begin{aligned}
\mathcal{M}^{-1} & =M^{-1}-M^{-1}(g \Gamma) M^{-1}, \\
g^{-1} & =1+\operatorname{Tr}\left(\Gamma M^{-1}\right) .
\end{aligned}
$$

The algorithm developed by Miller [34] for matrix inversion is analogous to the Sherman-Morrison-Woodbury formula that is better known to mathematicians and statisticians 35. We adopt here Miller's approach as its generalization to the addition of an arbitrary number of observables is relatively straightforward.

Having obtained the augmented covariance matrix, we now compute the improved theoretical uncertainty in the slope of the symmetry energy $L$ (a quantity that we denote with a "bar"). That is,

$$
\begin{aligned}
\bar{\tau}_{L}^{2} & =\sum_{i, j=1}^{F}\left(\frac{\partial L}{\partial p_{i}}\right)_{0} \mathcal{M}_{i j}^{-1}\left(\frac{\partial L}{\partial p_{j}}\right)_{0} \\
& =\tau_{L}^{2}-g \sum_{i, j=1}^{F}\left(\frac{\partial L}{\partial p_{i}}\right)_{0}\left(M^{-1} \Gamma M^{-1}\right)_{i j}\left(\frac{\partial L}{\partial p_{j}}\right)_{0} \\
& =\tau_{L}^{2}\left(1-g \frac{\tau_{I}^{2}}{\sigma_{I}^{2}} \varrho^{2}\left(L, \mathcal{O}_{I}\right)\right)
\end{aligned}
$$

where $g$ is given by

$$
g^{-1}=1+\operatorname{Tr}\left(\Gamma M^{-1}\right)=1+\frac{\tau_{I}^{2}}{\sigma_{I}^{2}} .
$$

In this way, one obtains the following simple and illuminating expression for the posterior variance of $L$ :

$$
\frac{\bar{\tau}_{L}^{2}}{\tau_{L}^{2}}=1-\frac{\varrho^{2}\left(L, \mathcal{O}_{I}\right)}{1+\sigma_{I}^{2} / \tau_{I}^{2}} \equiv 1-\alpha_{I}^{2} \varrho^{2}\left(L, \mathcal{O}_{I}\right) .
$$

Note that the sole experimental contribution to this expression is $\sigma_{I}$. Although many of the features encapsulated in the above expression are intuitive, the merit of the above expression is that it is quantitatively precise. Indeed, once the central assumption underlying this work has been adopted, no additional assumptions or approximations are required to obtain Eq.(11). In order to maximize the impact of the new measurement, the second term in Eq.(11) should be made as large as possible. Evidently, if the new observable is perfectly correlated (or anti-correlated) to $L$ and the experimental precision significantly improves on the current theoretical uncertainty, then the reduction in the uncertainty is maximized.

Information content of two new observables. Following the same exact procedure as before, one can write the augmented curvature matrix in the case that two new observables are added to the chi-square function. In this case the augmented curvature matrix is given by

$$
\mathcal{M}=M+\Gamma_{I}+\Gamma_{I I} \equiv \mathcal{M}_{I}+\Gamma_{I I},
$$

where $\Gamma_{I I}$ is the analog of Eq. (7) for the second observable $\mathcal{O}_{I I}$ and hence, a symmetric rank-1 matrix. Thus, the newly augmented matrix has the same structure as in Eq.(6), namely, a positive definite invertible matrix $\mathcal{M}_{I}$ plus a rank-1 matrix $\Gamma_{I I}$. This guarantees that the new curvature matrix is also invertible:

$$
\begin{aligned}
\mathcal{M}^{-1} & =\mathcal{M}_{I}^{-1}-\mathcal{M}_{I}^{-1}\left(\widetilde{g} \Gamma_{I I}\right) \mathcal{M}_{I}^{-1}, \\
\widetilde{g}^{-1} & =1+\operatorname{Tr}\left(\Gamma_{I I} \mathcal{M}_{I}^{-1}\right)
\end{aligned}
$$

Given that one is interested in assessing the impact of the two new measurements on prior theoretical uncertainties, one must compute the posterior covariance matrix $\mathcal{M}^{-1}$, not in terms of $\mathcal{M}_{I}^{-1}$ but rather, in terms of $M^{-1}$. Because of space limitations, we only present here the main results and postpone a detailed derivation to a forthcoming article. In terms of the original curvature matrix $M$ and the two additional rank-1 matrices $\Gamma_{I}$ and $\Gamma_{I I}$, the augmented covariance matrix is given by

$$
\mathcal{M}^{-1}=M^{-1}-M^{-1} \frac{\left[\left(\Omega_{I}+\Omega_{I I}\right)-\left(\Omega_{I} M^{-1} \Omega_{I I}+\Omega_{I I} M^{-1} \Omega_{I}\right)\right]}{1-\operatorname{Tr}\left(\Omega_{I} M^{-1} \Omega_{I I} M^{-1}\right)} M^{-1}
$$


where we have defined

$$
\Omega_{\lambda} \equiv g_{\lambda} \Gamma_{\lambda}=\frac{\Gamma_{\lambda}}{1+\operatorname{Tr}\left(\Gamma_{\lambda} M^{-1}\right)} ; \quad(\lambda=I, I I) .
$$

Following the same steps as in Eq.99, one can now assess the impact of the two new measurements on the theoretical uncertainty in $L$ :

$$
\frac{\bar{\tau}_{L}^{2}}{\tau_{L}^{2}}=1-\left[\frac{\alpha_{I}^{2} \varrho^{2}\left(L, \mathcal{O}_{I}\right)+\alpha_{I I}^{2} \varrho^{2}\left(L, \mathcal{O}_{I I}\right)-2 \alpha_{I}^{2} \alpha_{I I}^{2} \varrho\left(L, \mathcal{O}_{I}\right) \varrho\left(\mathcal{O}_{I}, \mathcal{O}_{I I}\right) \varrho\left(\mathcal{O}_{I I}, L\right)}{1-\alpha_{I}^{2} \alpha_{I I}^{2} \varrho^{2}\left(\mathcal{O}_{I}, \mathcal{O}_{I I}\right)}\right],
$$

where $\alpha_{\lambda}^{2}$ has been defined in Eq. 111). Besides the obvious generalization of the single term appearing in Eq. (11) to two new observables, there is an extra contribution from the correlation between the two new observables. In particular, if the two new observables are perfectly correlated, i.e., $\varrho\left(\mathcal{O}_{I}, \mathcal{O}_{I I}\right)=1$, then $\varrho\left(L, \mathcal{O}_{I}\right)=\varrho\left(L, \mathcal{O}_{I I}\right)$. Further, if the "first" observable is measured with high precision such that $\alpha_{I} \approx 1$, then

$$
\frac{\bar{\tau}_{L}^{2}}{\tau_{L}^{2}}=1-\varrho^{2}\left(L, \mathcal{O}_{I}\right)
$$

This expression is identical to Eq. 11) in the appropriate limit, so the "second" measurement becomes superfluous.

We have mentioned that the entire procedure hinges on the fact that the augmented curvature matrix may be written as the sum of an invertible curvature matrix plus a rank-1 matrix. This fact alone guarantees that the augmented curvature matrix is invertible. Thus, this recursive procedure can be generalized to an arbitrary number of new measurements [34, a topic that will be addressed in a forthcoming publication.

Results. Given that many critical quantities associated with the equation of state are not genuine physical observables, we now provide a couple of examples on how future experiments and observations may be used to improve the theoretical uncertainties in such quantities.

By the end of 2020 analyses of the PREX-II and CREX campaigns are expected to be completed. The expectation is that these experiments will reach a precision of $0.06 \mathrm{fm}$ for $R_{n}^{208}$ and of $0.03 \mathrm{fm}$ for the corresponding radius in ${ }^{48} \mathrm{Ca}$. Adopting these two experimental errors as the current theoretical uncertainties, we want to examine the impact on $L$ from MREX at MESA, which aims to determine $R_{n}^{208}$ and $R_{n}^{48}$ with a precision of $0.03 \mathrm{fm}$ and $0.02 \mathrm{fm}$, respectively [10]. To assess the impact of MREX one needs a model to connect the two physical observables $\left(R_{n}^{208}\right.$ and $\left.R_{n}^{48}\right)$ to the unobservable slope of the symmetry energy. To do so, we employ the covariant energy density functional FSUGold2 21] that was calibrated using exclusively well-measured observables, namely, ground-state binding energies and charge radii, centroid energies of giant monopole resonances, and observational limits on the maximum neutron star mass. FSUGold2 predicts the following correlation coefficients for the quantities of interest: $\varrho\left(R_{n}^{208}, R_{n}^{48}\right)=0.963$, $\varrho\left(L, R_{n}^{208}\right)=0.966$, and $\varrho\left(L, R_{n}^{48}\right)=0.921$. Using these predictions, one can now estimate the reduction in the theoretical uncertainty in $L$ from improved measurements of neutron radii. Results are displayed in Fig, 1 as a

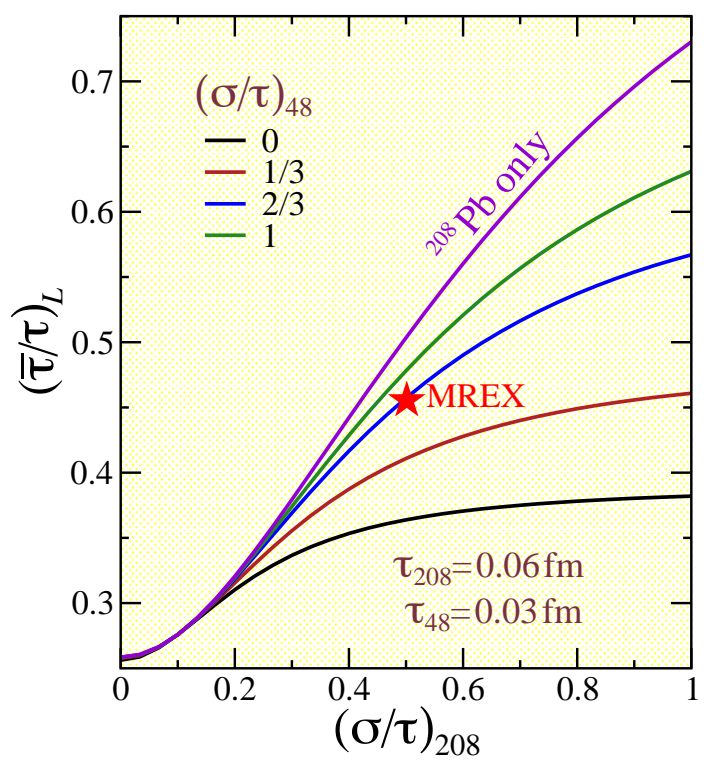

FIG. 1. (Color online) Reduction in the theoretical uncertainty of the slope of the symmetry energy $L$ as a consequence of improved measurements of the neutron radius of both ${ }^{208} \mathrm{~Pb}$ and ${ }^{48} \mathrm{Ca}$. The star in the figure reflects the impact of MREX on the slope of the symmetry energy.

function of the fractional experimental error $\left(\sigma_{208} / \tau_{208}\right)$ for a few values of the corresponding quantity in ${ }^{48} \mathrm{Ca}$. The "star" in the middle indicates the more than a factor of two improvement in the theoretical uncertainty in $L$ under the assumption that MREX reaches its precision goals. Also shown in the figure is the result obtained from assuming that only $R_{n}^{208}$ is measured. The figure suggests that one could achieve the projected MREX theoretical uncertainty in $L$ from a slightly improved single measurement of $R_{n}^{208}$. We underscore that although the numbers adopted for this example are representative of our current understanding, the main reason behind this - and the next example - is to illustrate the insights encapsulated in Eq.(16).

In the next example we illustrate the impact of new experiments and observations on another quantity that, while critical to our understanding of neutron-rich matter, is not accessible to experiment: the pressure of pure neutron matter at two times saturation density $P_{2}$. 
Twice nuclear matter saturation density provides a powerful bridge between terrestrial experiments and cosmological observations. At terrestrial facilities neutron-rich matter at above saturation density can be probed via energetic collisions of heavy ions with a large neutronproton asymmetry $9,10,37,39$. In the cosmos, the pressure of neutron-rich matter at supra saturation density can be elucidated from the structure of neutron stars. Recent detections by both the LIGO-Virgo and NICER collaborations have started to provide constraints on the compactness of neutron stars and ultimately on stellar radii [40 50]. In turn, stellar radii seem to be strongly correlated to the pressure of pure neutron matter at twoto-three times saturation density [51 54. For a comprehensive recent review on equation of state constraints see Ref. [55] and references contained therein.

In Fig 2 we display the reduction in the theoretical uncertainty in the pressure of pure neutron matter at twice saturation density $\left(P_{2}\right)$ as a consequence of improved measurements of stellar radii and neutron skins. The theoretical uncertainty in the radius of a $1.4 M_{\odot}$ neutron star is set to $1.2 \mathrm{~km} \mathrm{6,} \mathrm{7].} \mathrm{As} \mathrm{done} \mathrm{earlier,} \mathrm{we} \mathrm{used} \mathrm{pre-}$ dictions from FSUGold2 for the correlation coefficients of interest: $\varrho\left(R_{1.4}, R_{n}^{208}\right)=0.968, \varrho\left(P_{2}, R_{1.4}\right)=0.996$, and $\varrho\left(P_{2}, R_{n}^{208}\right)=0.966$. Figure 2 shares many similar

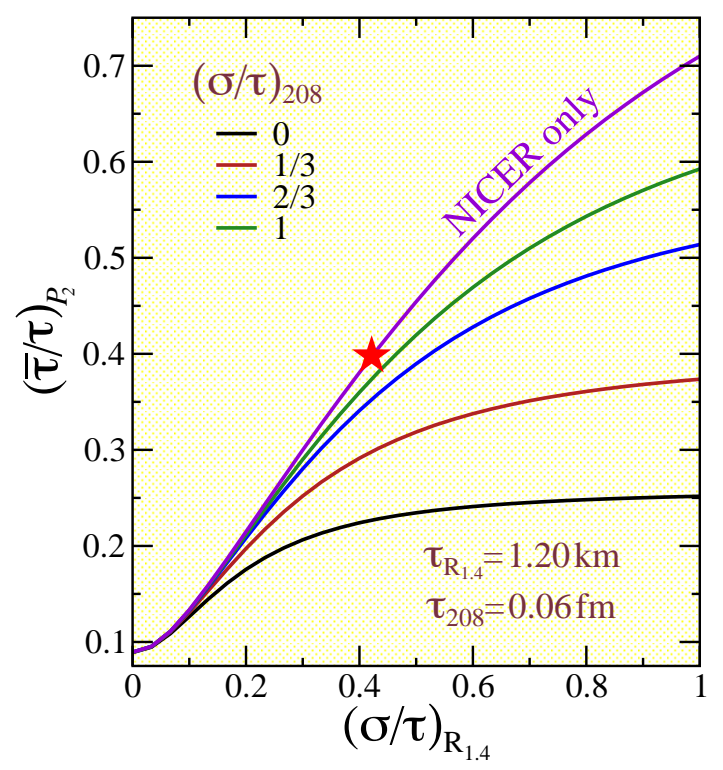

FIG. 2. (Color online) Reduction in the theoretical uncertainty of the pressure of pure neutron matter at twice nuclear matter saturation density as a consequence of improved measurements of the radius of a $1.4 M_{\odot}$ neutron star and the neutron radius of ${ }^{208} \mathrm{~Pb}$. The star in the figure reflects the impact of a $0.5 \mathrm{~km}$ measurement of the radius of J0437-4715.

features to Fig1. However, the slightly larger correlation coefficients suggest that a "perfect" measurement of the stellar radius could reduce the theoretical uncertainty in $P_{2}$, by nearly $90 \%$. The star in the figure represents the impact of a $\pm 0.5 \mathrm{~km}$ determination of the stellar radius of J0437-471 [6, 7], a neutron star with a well determined mass of $1.44(7) M_{\odot}[56$. By itself, this measurement would reduce the theoretical uncertainty in $P_{2}$ by about $60 \%$.

We close this section with a word of caution. Whereas the strong correlation between the neutron skin thickness of ${ }^{208} \mathrm{~Pb}$ and $L$ has been firmly established [27, we find that the correlation between the radius of a $1.4 M_{\odot}$ neutron star and the pressure of pure neutron matter at twice saturation density is model dependent. Indeed, accurately calibrated models with a soft symmetry energy, such as FSUGarnet [57, predict a correlation coefficient of only $\varrho\left(P_{2}, R_{1.4}\right)=0.4$, even if the entire equation of state - containing contributions from both symmetric nuclear matter and the symmetry energy - is stiff enough to support two solar-mass neutron stars.

Conclusions. Motivated by one of the central questions posed in Ref.[13], namely, "How can new data reduce uncertainties of current theoretical models?", we developed a fully analytic approach to answer this question. Our entire formalism hinges on one underlying assumption, namely, that the location of the minimum of the chi-square function remains unchanged after incorporating the new set of observables. Once this assumption is adopted, simple and insightful expressions were developed as one or two observables are added to the calibration of the model parameters. Precise details on the derivation of our main results, encapsulated in Eqs.(11) and (16), and their generalization to the case of an arbitrary number of new observables will be presented in a longer forthcoming publication.

Given the relative simplicity of Eqs.(11) and (16) we suspect that such expressions are well known to mathematicians and statisticians. Yet despite our best effortsand that of several colleagues - we could not find such a result in the literature. Although we found countless applications of the Sherman-Morrison-Woodbury formula, we found no answer to the question of how can new data reduce uncertainties of current theoretical models.

Acknowledgments. We thank Pablo Giuliani for a careful reading of the manuscript and for many useful comments. We also thank Jonathan Bradley and Antonio Linero for many useful conversations. This material is based upon work supported by the U.S. Department of Energy Office of Science, Office of Nuclear Physics under Award Number DE-FG02-92ER40750 . 
[1] B. P. Abbott et al. (Virgo, LIGO Scientific), Phys. Rev. Lett. 119, 161101 (2017).

[2] M. R. Drout et al., Science 358, 1570 (2017)

[3] D. Geesaman et al., "Reaching for the horizon; the 2015 long range plan for nuclear science,".

[4] Connecting Quarks with the Cosmos: Eleven Science Questions for the New Century (The National Academies Press, Washington, 2003).

[5] H. T. Cromartie et al., Nat. Astron. 4, 72 (2019).

[6] M. C. Miller et al., Astrophys. J. Lett. 887, L24 (2019).

[7] T. E. Riley et al., Astrophys. J. Lett. 887, L21 (2019).

[8] M. Tsang, J. Stone, F. Camera, P. Danielewicz, S. Gandolfi, et al., Phys.Rev. C86, 015803 (2012).

[9] C. J. Horowitz, E. F. Brown, Y. Kim, W. G. Lynch, R. Michaels, et al., J. Phys. G41, 093001 (2014).

[10] M. Thiel, C. Sfienti, J. Piekarewicz, C. J. Horowitz, and M. Vanderhaeghen, J. Phys. G46, 093003 (2019).

[11] PRA-Editors, Phys. Rev. A 83, 040001 (2011).

[12] M. Kortelainen, T. Lesinski, J. More, W. Nazarewicz, J. Sarich, et al., Phys. Rev. C82, 024313 (2010).

[13] P.-G. Reinhard and W. Nazarewicz, Phys. Rev. C81, 051303(R) (2010)

[14] F. Fattoyev and J. Piekarewicz, Phys. Rev. C84, 064302 (2011).

[15] F. Fattoyev and J. Piekarewicz, Phys. Rev. C 86, 015802 (2012)

[16] J. Piekarewicz, B. Agrawal, G. Colò, W. Nazarewicz, N. Paar, et al., Phys. Rev. C85, 041302(R) (2012).

[17] P.-G. Reinhard and W. Nazarewicz, Phys. Rev. C87, $014324(2013)$

[18] J. Erler, C. J. Horowitz, W. Nazarewicz, M. Rafalski, and P.-G. Reinhard, Phys. Rev. C87, 044320 (2013).

[19] F. Fattoyev, W. Newton, and B.-A. Li, Phys. Rev. C 90, 022801 (2014)

[20] J. Dobaczewski, W. Nazarewicz, and P.-G. Reinhard, J. Phys. G41, 074001 (2014)

[21] W.-C. Chen and J. Piekarewicz, Phys. Rev. C90, 044305 (2014).

[22] D. G. Ireland and W. Nazarewicz, J. Phys. G42, 030301 (2015).

[23] J. Piekarewicz, A. R. Linero, P. Giuliani, and E. Chicken, Phys. Rev. C94, 034316 (2016)

[24] B. A. Brown, Phys. Rev. Lett. 85, 5296 (2000).

[25] R. J. Furnstahl, Nucl. Phys. A706, 85 (2002).

[26] M. Centelles, X. Roca-Maza, X. Viñas, and M. Warda, Phys. Rev. Lett. 102, 122502 (2009).

[27] X. Roca-Maza, M. Centelles, X. Viñas, and M. Warda, Phys. Rev. Lett. 106, 252501 (2011).

[28] S. Abrahamyan, Z. Ahmed, H. Albataineh, K. Aniol, D. S. Armstrong, et al., Phys. Rev. Lett. 108, 112502 (2012).

[29] C. J. Horowitz, Z. Ahmed, C. M. Jen, A. Rakhman, P. A. Souder, et al., Phys. Rev. C85, 032501 (2012).

[30] "CREX: Parity-violating measurement of the weak charge distribution of ${ }^{48} \mathrm{Ca}$," http://hallaweb.jlab. org/parity/prex/c-rex/c-rex.pdf.
[31] P. C. Gregory, "Bayesian logical data analysis for the physical sciences," (Cambridge University Press, Cambridge, UK, 2005).

[32] J. V. Stone, "Bayes' rule: A tutorial introduction to bayesian analysis," (Sebtel Press, Sheffield, UK, 2013).

[33] J. Piekarewicz, W.-C. Chen, and F. Fattoyev, J. Phys. G42, 034018 (2015).

[34] K. S. Miller, Mathematics Magazine 54, 67 (1981).

[35] S. Banerjee and A. Roy, "Linear algebra and matrix analysis for statistics," (CRC Press, Boca Raton, FL, 2014).

[36] D. Becker et al., (2018), 10.1140/epja/i2018-12611-6. arXiv:1802.04759 [nucl-ex]

[37] M. B. Tsang et al., Phys. Rev. Lett. 102, 122701 (2009)

[38] M. Tsang, W. Lynch, P. Danielewicz, and C. Tsang, Phys. Lett. B 795, 533 (2019)

[39] "FRIB400: The Scientific Case for the $400 \mathrm{MeV} / \mathrm{u}$ Energy Upgrade of FRIB," https://fribusers.org/ documents/2019/FRIB400-Upgrade.pdf.

[40] A. Bauswein, O. Just, H.-T. Janka, and N. Stergioulas, Astrophys. J. 850, L34 (2017).

[41] F. J. Fattoyev, J. Piekarewicz, and C. J. Horowitz, Phys. Rev. Lett. 120, 172702 (2018)

[42] E. Annala, T. Gorda, A. Kurkela, and A. Vuorinen, Phys. Rev. Lett. 120, 172703 (2018)

[43] B. P. Abbott et al. (Virgo, LIGO Scientific), Phys. Rev. Lett. 121, 161101 (2018)

[44] E. R. Most, L. R. Weih, L. Rezzolla, and J. SchaffnerBielich, Phys. Rev. Lett. 120, 261103 (2018).

[45] I. Tews, J. Margueron, and S. Reddy, Phys. Rev. C98, $045804(2018)$

[46] T. Malik, N. Alam, M. Fortin, C. Providencia, B. K. Agrawal, T. K. Jha, B. Kumar, and S. K. Patra, Phys. Rev. C98, 035804 (2018)

[47] C. Y. Tsang, M. B. Tsang, P. Danielewicz, W. G. Lynch, and F. J. Fattoyev, (2018), arXiv:1807.06571 [nucl-ex],

[48] D. Radice, A. Perego, F. Zappa, and S. Bernuzzi, Astrophys. J. Lett. 852, L29 (2018).

[49] D. Radice and L. Dai, Eur. Phys. J. A 55, 50 (2019).

[50] W.-J. Xie and B.-A. Li, (2020), arXiv:2005.07216 [astroph.HE]

[51] J. M. Lattimer and M. Prakash, Phys. Rept. 442, 109 (2007).

[52] I. Tews, J. Margueron, and S. Reddy, Eur. Phys. J. A 55, 97 (2019).

[53] C. D. Capano, I. Tews, S. M. Brown, B. Margalit, S. De, S. Kumar, D. A. Brown, B. Krishnan, and S. Reddy, Nature Astronomy 4, 625 (2019)

[54] C. Drischler, J. Melendez, R. Furnstahl, and D. Phillips, (2020), arXiv:2004.07805 [nucl-th].

[55] K. Chatziioannou, (2020), arXiv:2006.03168 [gr-qc]

[56] D. Reardon et al., Mon. Not. Roy. Astron. Soc. 455, 1751 (2016).

[57] W.-C. Chen and J. Piekarewicz, Phys. Lett. B748, 284 (2015). 